\title{
D
}

'Educational Activities, European Respiratory Society, Lausanne, Switzerland. '2Dept of Pulmonology, Gentofte Hospital, University of Copenhagen, Copenhagen, Denmark. ${ }^{3}$ Dept of Pneumology and Critical Care Medicine, Thoraxklinik, University of Heidelberg. Heidelberg. Germany. ${ }^{4}$ Copenhagen University and the Capital Region of Denmark Centre for Clinical Education, University of Copenhagen, Copenhagen, Denmark. ${ }^{5}$ Dept of Respiratory Medicine, Maastricht University Medical Center, Maastricht, The Netherlands. ${ }^{6}$ Dept of Respiratory Medicine, Academic Medical Centre, University of Amsterdam, Amsterdam, The Netherlands.

\section{Endobronchial ultrasound: launch of an ERS structured training programme}

\section{Introduction}

The systematic assessment of mediastinal anatomy and in particular lymph nodes gains increasing importance in malignant as well as in non-malignant diseases. However, the diagnosis and staging of lung cancer is the most frequent indication. Lung cancer is the fourth most prevalent form of cancer in Europe, with lung cancer making up the largest number of cancer-related deaths [1]. Between European countries there are variations in the incidence and mortality rates of lung cancer. These are reflected by differences in national health system policies, regional risk factors, disparities in human development and local cancer control measures [1]. International health systems are looking for more cost-effective and accurate tools for screening and diagnosing lung cancer and other respiratory diseases.

One such procedure that is helping clinicians in daily practice is endobronchial ultrasound (EBUS). A relatively new technique within the field of interventional pulmonology, EBUS is a minimally invasive procedure that can be used on an outpatient basis to not only diagnose and stage lung cancer but also to assess mediastinal nodes for other diseases such as sarcoidosis, tuberculosis or metastases from extrathoracic malignancies. Previously, more invasive and costly procedures such as mediastinoscopy were required to reach the same diagnosis. The EBUS technique uses ultrasound along with a bronchoscope to visualise the bronchial wall and the adjacent mediastinal structures, meanwhile enabling real-time ultrasound-guided aspirations.

As with all procedures, the success of the procedure is dependent on the skill level of the operator. It has been found that learning this technique has a significant learning curve [2]. Although, it has been internationally recognised that the "see one, do one" form of training is not optimal for teaching complex clinical procedures, there is currently no European or international consensus on how training should be delivered to ensure that physicians are competent in this technique and well-equipped to perform on patients [2, 3]. Up until now there have been several studies that outline the benefit of structured simulation training in a physician's training schedule, and most notably for this programme there is the EBUSAT framework, a validated approach to assessing EBUS performance [2, 4].

Consequently in September 2014, a task force of EBUS experts was mobilised from three university hospitals (Heidelberg, Copenhagen and Amsterdam) and an expert simulation centre to investigate, formulate and implement a comprehensive training programme to train qualified
Cite as: Farr A, Clementsen $P$, Herth F, et al. Endobronchial ultrasound: launch of an ERS structured training programme. Breathe 2016; 12: 217-220. 


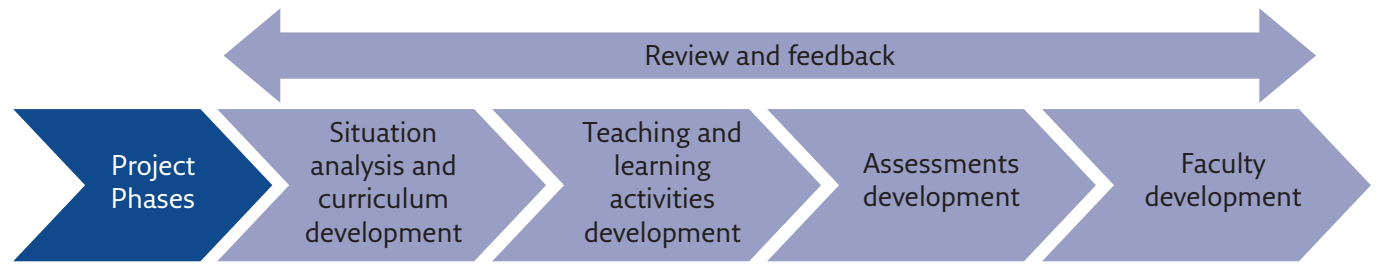

Figure 1 Development phases for the EBUS structured training programme.

doctors to be able to independently and competently perform EBUS. Following a similar approach as the ERS Spirometry training programme, the project was split into a number of development phases (figure 1) [5].

This article will discuss the first phase, the situation analysis and the curriculum development, and will provide an overview of the programme.

\section{Methods}

The approach, methods and processes used in this project have been adapted from the ERS developed educational harmonisation framework, that was used in the flagship adult and paediatric medicine projects and eight other post-specialty programmes since [6, 7].

\section{Situation analysis and curriculum development}

Following the process of ERS educational curricula developed thus far, the EBUS task force identified

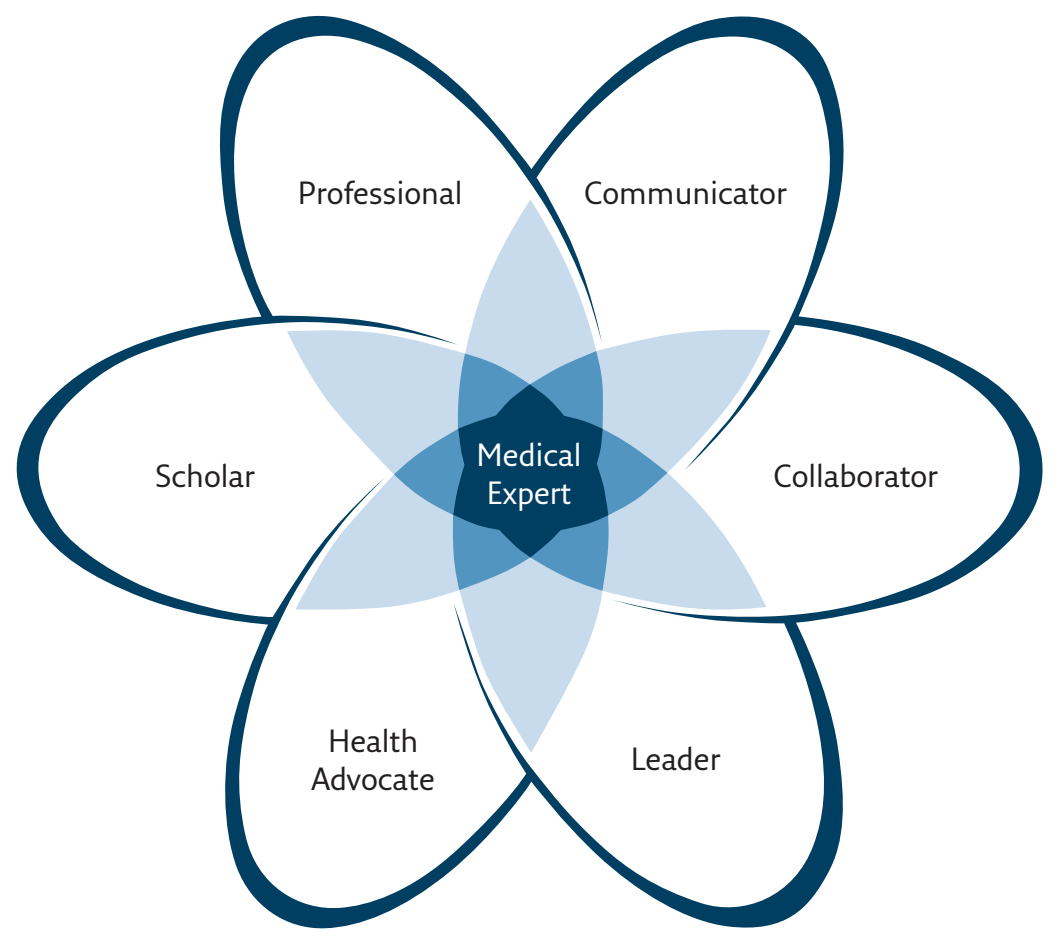

Figure 2 The CanMEDS framework. (c) 2015 The Royal College of Physicians and Surgeons of Canada. www.royalcollege.ca/rcsite/canmeds/canmeds-framework-e Reproduced with permission. the current situation of EBUS training, set the target audience and defined objectives for the programme.

To gather information about current training opportunities an internet search using the keywords EBUS and endobronchial ultrasound was completed in German, English and French to identify courses, online learning, fellowships and guidelines openly available on the internet. 15 activities were identified. From these activities key topics and formats were extracted and were used as a basis for the syllabus document.

The next step was to define the target audience for this training programme. Undeniably the EBUS procedure and the diagnosis of lung cancer in general are performed in a multidisciplinary environment [8-10]. There was much discussion within the task force around this point. Ideally the training programme would offer learning opportunities for all team members; however, many challenges were raised about the feasibility and cost of developing such a programme for all medical professionals involved in performing an EBUS procedure. It was decided in the first stage that this programme would focus on the operator, the professional that is responsible for the manoeuvring the scope during the procedure. In current practice in Europe, it is a medical doctor that performs this role. Therefore the target audience was defined as qualified medical doctors with previous experience in: performing bronchoscopies; TNM (tumour, node, metastasis) classification of lung cancer; and staging of oncology patients and other conditions.

In order to ensure that the training programme content was robust, fit for an international audience and free from individual opinion, it was decided to develop a consensusbased curriculum. A curriculum defines the learning that is expected to take place during the training programme, with the learning defined in terms of knowledge, skills and attitudes [11]. ERS curricula also outline the teaching and learning activities and assessments. To ensure the international perspective was considered the curriculum was developed by four experts from three countries. In addition, a group of nine international EBUS experts, including experts from Asia and the USA, were identified to act as independent content reviewers.

The syllabus provides a clear foundation to allow the curriculum to be constructed. Based on the information gathered through the situation 
analysis an initial list of 50 topics was discussed and refined through several stages and series of independent review until a list of 19 key topics was identified. Rather than separating the items into larger modules largely driven by pathophysiology, diagnosis, treatment and management as is seen in other ERS curricula, it was decided to organise this curriculum into modules based on the stages of clinical practice: pre-procedure, procedure and post-procedure.

The next step in the curriculum development was to detail the key competencies required within each syllabus item, which can be seen represented within the curriculum document as the knowledge, skills and attitudes. ERS uses the CanMEDs framework as a guide when developing these key competencies (figure 2). This framework identifies seven roles for medical professionals: medical expert, communicator, collaborator, health advocate, leader, scholar and professional [12]. The CanMEDS framework was developed in response to societal demand for greater accountability and professionalism in medicine.

Consequently, these roles are included in the training programme to ensure that learners will be well-equipped to provide a high level of patient care with a dedication to professionalism. Full details of the curriculum can be found in the supplementary material.

\section{Overview of the programme}

As mentioned previously, the EBUS procedure is a skill with a long learning curve. To allow participants significant time to develop this new acquired skill, the task force carefully considered the most comprehensive way to guide the participants through the learning process. It was decided to develop the training programme in a modular format, for consistency with other ERS programmes these modules were named parts. The parts were developed based on the Miller's Model of clinical practice (figure 3) [13].

Part 1 requires participants to follow seven online self-directed modules comprised of webcasts, selfassessment quizzes and interactive maps. Next participants attend a physical course where the content is revised, EBUS equipment introduced and a demonstration of live procedures is performed. After the course, participants have 2 weeks to revise all material covered prior to sitting an online multiple-choice question assessment. Part 2 comprises of intensive simulation-based training and active clinical observation, with both activities being assessed. Part 3 sees participants starting to perform procedures in a supervised environment, participants will be asked to document procedures in an online portfolio. Finally, the participant, with support from the host supervisor, can then submit 20 written reports and three video procedures for assessment. The complete training programme is

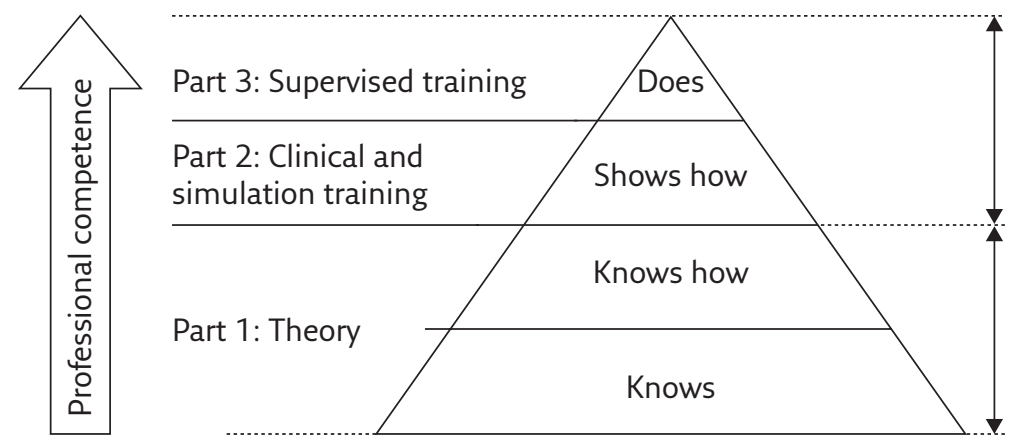

Figure 3 Miller's model of clinical competence. Reproduced from [13] with permission.

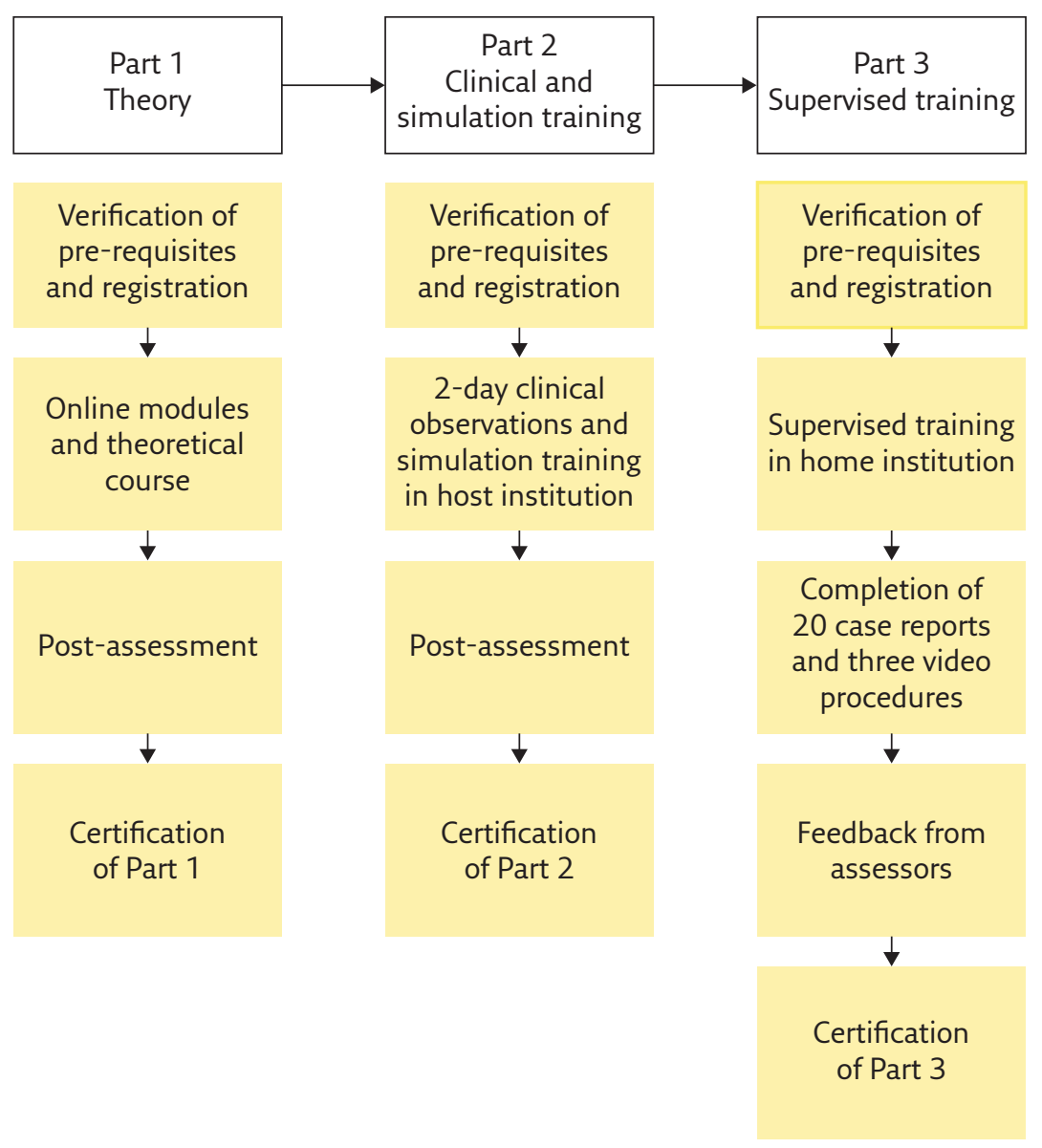

Figure 4 The complete ERS EBUS training programme.

illustrated in figure 4 . All three parts of the training programme need to be successfully completed for the participant to receive the ERS EBUS certification.

\section{Conclusion and next steps}

The EBUS task force will strive to improve the programme by incorporating continuous feedback from experts and programme participants from the teaching and learning activities and assessments, developing future trainers and EBUS experts to ensure the longevity of the 
training programme, and look at the feasibility of expanding into other countries and regions with the ultimate aim of improving the success of this technique in clinical practice.

Further details regarding the ERS EBUS training programme can be found on the ERS website: www.ersnet.org/ebus

\section{Acknowledgements}

ERS would like to thank the ERS EBUS task force, independent reviewers and all of the teaching faculty. The conception and development of this project is thanks to this very dedicated and voluntary group of international interventional pulmonology experts.

\section{Supplementary material}

This article has supplementary material available from breathe.ersjournals.com

\section{Conflict of interest}

A. Farr and S. Dowsland are employees of the European Respiratory Society.

\section{References}

1. Ferlay J, Steliarova-Foucher E, Lortet-Tieulent J, et al. Cance incidence and mortality patterns in Europe: estimates for 40 countries in 2012. EurJ Cancer 2013; 49: 1374-1403.

2. Konge L, Clementsen PF, Ringsted C, et al. Simulator training for endobronchial ultrasound: a randomised controlled trial. Eur Respir J 2015; 46: 1140-1149.

3. Grantcharov TP, Reznick RK. Teaching procedural skills. BM 2008; 336: 1129-1131.

4. Konge L, Annema J, Clementsen P, et al. Using virtual-reality simulation to assess performance in endobronchial ultrasound. Respiration 2013; 86: 59-65.

5. Cooper B, Steenbruggen I, Mitchell S, et al. Hermes spirometry: the European spirometry driving licence. Breathe 2011; 7: 258-275

6. Loddenkemper R, Haslam P, Séverin T, et al. European curriculum recommendations for training in adult respiratory medicine. Breathe 2008; 5: 80-93.

7. Gappa M, Noël J-L, Séverin T, et al. Paediatric HERMES: European curriculum recommendations for training in paediatric respiratory medicine. Breathe 2010; 7: 72-117.
8. Herbert A, Santis G. EBUS-TBNA: an opportunity for clinicians, cytopathologists and patients to gain from multidisciplinary collaboration. Cytopathology 2010; 21: 3-5.

9. Goller S. Combined EUS and EBUS procedures: a multidisciplinary approach to patient care. Gastroenterol Nurs 2008; 31: 164.

10. Paiano S, Bertolaccini L, Pomari C, et al. EBUS TBNA negative lymph nodes risk stratification model: a tool for multidisciplinary team. Eur Respir J 2015; 46: Suppl. 59, OA1745

11. McKimm J, Swanwick T. Clinical Teaching Made Easy: A Practical Guide to Teaching and Learning in Clinical Settings. London, Andrews UK Limited, 2013.

12. Frank JR, Danoff D. The CanMEDS initiative: implementing an outcomes-based framework of physician competencies. Med Teach 2007; 29: 642-647.

13. Miller GE. The assessment of clinical skills/ competence/performance. Acad Med 1990; 65: Suppl., S63-S67. 